Article

\title{
A Convenient Ultrasound-Promoted Synthesis of Some New Thiazole Derivatives Bearing a Coumarin Nucleus and Their Cytotoxic Activity
}

\author{
Sobhi M. Gomha ${ }^{1, *}$ and Khaled D. Khalil ${ }^{1,2}$ \\ 1 Department of Chemistry, Faculty of Science, University of Cairo, Giza 12613, Egypt \\ 2 Chemistry Department, Faculty of Science, University of Kuwait, P.O. Box 5969, Safat 13060, \\ Kuwait; E-Mail: khd.khalil@yahoo.com \\ * Author to whom correspondence should be addressed; E-Mail: s.m.gomha@hotmail.com; \\ Tel.: +20-237-400-304; Fax: +20-225-685-799.
}

Received: 25 June 2012; in revised form: 18 July 2012 / Accepted: 24 July 2012 /

Published: 3 August 2012

\begin{abstract}
Successful implementation of ultrasound irradiation for the rapid synthesis of a novel series of 3-[1-(4-substituted-5-(aryldiazenyl)thiazol-2-yl)hydrazono)ethyl]-2Hchromen-2-ones $\mathbf{5 a}-\mathbf{h}$, via reactions of 2-(1-(2-oxo-2H-chromen-3-yl)ethylidene) thiosemicarbazide (2) and the hydrazonoyl halides 3(4), was demonstrated. Also, a new series of 5-arylidene-2-(2-(1-(2-oxo-2H-chromen-3-yl)ethylidene)hydrazinyl)thiazol$4(5 H)$-ones 10a-d were synthesized from reaction of $\mathbf{2}$ with chloroacetic acid and different aldehydes. Moreover, reaction of 2-cyano- $N^{\prime}$-(1-(2-oxo- $2 H$-chromen-3-yl)ethylidene)acetohydrazide (12) with substituted benzaldehydes gave the respective arylidene derivatives 13a-c under the conditions employed. The structures of the synthesized compounds were assigned based on elemental analyses and spectral data. Also, the cytototoxic activities of the thiazole derivative 5a was evaluated against $\mathrm{HaCaT}$ cells (human keratinocytes). It was found that compound $\mathbf{5 a}$ possess potent cytotoxic activity.
\end{abstract}

Keywords: thiosemicarbazides; thiazoles; hydrazonoyl halides; ultra-sound irradiation; cytotoxic activity 


\section{Introduction}

The synthesis of coumarins and their derivatives has attracted considerable attention from organic and medicinal chemists for many years as a large number of natural and synthetic products contain this heterocyclic nucleus [1-5]. A number of natural and synthetic coumarin derivatives have been reported to exert notable antimicrobial [1,2], antifungal [3,4] and cytotoxic [5] activity.

Thiazole derivatives have also attracted increasing attention due to their numerous pharmacological applications and biological activities, such as anti-inflammatory, analgesic, antimicrobial, anti-HIV, antihypertensive and herbicidal activity [6-10].

Ultrasonic-assisted organic synthesis (UAOS) is a powerful and green approach which is being used more and more to accelerate synthesis of organic compounds [11]. Increases in reaction rate and yields occur on application of ultrasound waves [12-16].

In view of these observations and in continuation of our previous work on the synthesis of heterocyclic systems for biological evaluation [17-19], we report herein a facile route to various thiazole derivatives incorporating coumarin moieties using the ultra-sound irradiation technique. Additionally we have found that one of the synthesized compounds has shown high cytotoxic activity.

\section{Results and Discussion}

\subsection{Chemistry}

2-(1-(2-Oxo-2H-chromen-3-yl)ethylidene)thiosemicarbazide (2) was previously prepared by refluxing 3-acetyl-2H-chromen-2-one (1) and thiosemicarbazide in absolute ethanol in the presence of catalytic amounts of $\mathrm{HCl}$ [20] (Scheme 1).

The target compounds 5a-h, 3-[1-(4-substituted-5-(aryldiazenyl)thiazol-2-yl)hydrazono) ethyl]-2Hchromen-2-ones, were synthesized in a one pot reaction of thiosemicarbazide $\mathbf{2}$ and hydrazonoyl halides 3(4) in the presence of TEA under ultrasonic irradiation (Scheme 1).

The structural elucidation of the compounds was based on spectral evidence and microanalyses. The mass spectra of these products 5a-c showed the molecular ion peaks at the expected $\mathrm{m} / \mathrm{z}$ values. Their IR spectra showed the disappearance of the $\mathrm{NH}_{2}$ group, and revealed in each case one band at $1568-1558 \mathrm{~cm}^{-1}$, assignable to the $\mathrm{N}=\mathrm{N}$ group (see Experimental).

The thiazole derivatives $\mathbf{8}(\mathbf{9})$ were synthesized in good yields by the treatment of thiosemicarbazide derivative 2 with chloroacetone (6) or phenacyl bromide (7) in dioxane under ultrasonic irradiation following the Hantzsch thiazole synthesis [21]. Upon coupling the thiazole derivatives 8(9) with diazotized aniline, in presence of sodium acetate trihydrate, the azo derivatives $\mathbf{5 a}-\mathbf{h}$ were obtained. The structures of the latter products were confirmed by the appearance of a $\mathrm{N}=\mathrm{N}$ band in the IR spectra and the lack of signals due to the C-5 proton of the thiazole ring in their ${ }^{1} \mathrm{H}-\mathrm{NMR}$ spectra (see Experimental). The azo derivatives of similar thiazoles have found wide applications in the dyeing of synthetic fibers [22,23] and the azo derivatives described in the present work may find similar applications.

4-Thiazolidinone compound $\mathbf{1 1}$ was obtained by reaction of thiosemicarbazide $\mathbf{2}$ with chloroacetic acid in glacial acetic acid and in the presence of anhydrous sodium acetate. Reaction of the latter product 11 with substituted aldehydes afforded the corresponding arylidines 10a-d. 
Scheme 1. Synthesis of 5-arylazothiazole derivatives $\mathbf{5 a}-\mathbf{h}$.<smiles>CC(=O)c1cc2ccccc2oc1=O</smiles><smiles>C/C(=N\NC(N)=S)c1cc2ccccc2oc1=O</smiles><smiles>[R]C(=O)NN</smiles>
3(4)

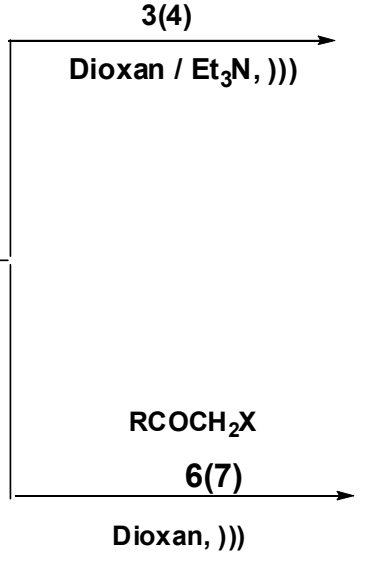

3,6 and 8: $\mathrm{R}=\mathrm{CH}_{3}, \quad \mathrm{X}=\mathrm{Cl}$; 4,7 and 9: $\mathrm{R}=\mathrm{C}_{6} \mathrm{H}_{5}, \quad \mathrm{X}=\mathrm{Br}$<smiles>[R]c1nc(N/N=C(\C)c2cc3ccccc3oc2=O)sc1N=[W]</smiles>

5a-h

的 $\mathrm{CH}_{3} \mathrm{COONa} 3 \mathrm{H}_{2} \mathrm{O}$

2

\begin{tabular}{|c|l|l|}
\hline 5a-h & $\mathbf{R}$ & $\mathbf{A r}$ \\
\hline a & $\mathrm{CH}_{3}$ & $\mathrm{C}_{6} \mathrm{H}_{5}$ \\
b & $\mathrm{CH}_{3}$ & $4-\mathrm{MeC}_{6} \mathrm{H}_{4}$ \\
c & $\mathrm{CH}_{3}$ & $4-\mathrm{ClC}_{6} \mathrm{H}_{4}$ \\
d & $\mathrm{CH}_{3}$ & $4-\mathrm{NO}_{2} \mathrm{C}_{6} \mathrm{H}_{4}$ \\
e & $\mathrm{Ph}$ & $\mathrm{C}_{6} \mathrm{H}_{5}$ \\
f & $\mathrm{Ph}$ & $4-\mathrm{MeC}_{6} \mathrm{H}_{4}$ \\
g & $\mathrm{Ph}$ & $4-\mathrm{ClC}_{6} \mathrm{H}_{4}$ \\
h & $\mathrm{Ph}$ & $4-\mathrm{NO}_{2} \mathrm{C}_{6} \mathrm{H}_{4}$ \\
& & \\
\hline
\end{tabular}<smiles>[R]c1csc(N/N=C(\C)c2cc3ccccc3oc2=O)n1</smiles>

8(9)

The one pot synthesis of products $\mathbf{1 0 a}-\mathbf{d}$ has been carried out via reaction of thiosemicarbazide $\mathbf{2}$ with chloroacetic acid and aldehydes in glacial acetic acid in presence of excess anhydrous sodium acetate (Scheme 2). The ${ }^{1} \mathrm{H}-\mathrm{NMR}$ spectra data were also consistent with the assigned structures; thiazolidinone $\mathrm{CH}_{2}$ protons of $\mathbf{1 1}$ appeared at $\delta 3.97 \mathrm{ppm}$, arylidiene $\mathrm{CH}$ proton of $\mathbf{1 0 a}-\mathbf{d}$ was observed at 8.60-8.67 ppm (see Experimental).

In addition, the hydrazide-hydrazone derivative 12 was prepared by ultrasonic irradiation of 3-acetyl-2H-chromen-2-one (1) and 2-cyanoacetohydrazide in absolute ethanol in the presence of catalytic amounts of $\mathrm{HCl}$ (Scheme 3). The structure of compound 12 was established on the basis of analytical and spectral data. Thus its ${ }^{1} \mathrm{H}-\mathrm{NMR}$ spectrum showed the presence of a singlet at $\delta 4.25 \mathrm{ppm}$ for the $\mathrm{CH}_{2}$ group, and a singlet at $\delta 11.19$ ppm for an $\mathrm{NH}$ group. Its mass spectrum revealed a molecular ion peak at $m / z 269$ (see Experimental). 
Scheme 2. Synthesis of 5-arylidenethiazolinone derivatives 10a-d.<smiles>C/C(=N\NC(N)=S)c1cc2ccccc2oc1=O</smiles>
AcOH ( $\mathrm{AcONa}$, )))<smiles>C/C(=N\NC1=NC(=O)CS1)c1cc2ccccc2oc1=O</smiles>
ArCHO

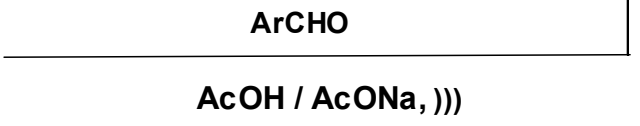

$$
\mathrm{Ar}=\mathrm{YC}_{6} \mathrm{H}_{4} ; \mathrm{Y}: \mathrm{a}, \mathrm{H} ; \quad \text { b, } \mathrm{CH}_{3} ; \mathrm{c}, 4-\mathrm{Cl} ; \mathrm{d}, 4-\mathrm{NO}_{2} \text {. }
$$

Scheme 3. Reaction of acetohydrazide 12 with aromatic aldehydes.<smiles>CC(=O)c1cc2ccccc2oc1=O</smiles>

1

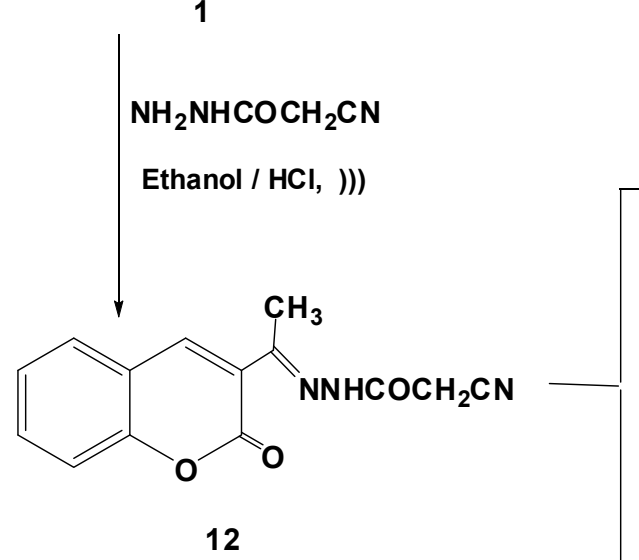

ArCHO EtOH, Pipridine, )))<smiles>C/C(=N\NC(=O)/C=C/Br)c1cc2ccccc2oc1=O</smiles>

13a-c $\mathrm{Ar}=\mathrm{YC}_{6} \mathrm{H}_{4} ; \mathrm{Y}: \mathrm{a}, \mathrm{H} ; \mathrm{b}, \mathrm{CH}_{3} ; \mathrm{c}, 4-\mathrm{Cl}$.<smiles>CCOc1cccc2oc(=O)c(C(C)=NNc3ccccc3)cc12</smiles>

Furthermore, treatment of the acetohydrazide 12 with substituted benzaldehydes, under ultrasonic irradiation, afforded the benzylidene derivatives 13a-c on the basis of their spectral data (Scheme 3) which confirmed the structures of the products by the appearance of a $\mathrm{C}=\mathrm{CH}$ signal at $\delta 9.34 \mathrm{ppm}$ and the lack of the characteristic signal due to methylene protons (see Experimental). The reaction of 12 with salicylaldehyde gave the coumarin derivative 14 (Scheme 3), in analogy with the reported literature [24,25]. The IR spectrum of compound 14 showed the lack of absorption bands corresponding to a $\mathrm{C} \equiv \mathrm{N}$ group and presence of bands at $3,198 \mathrm{~cm}^{-1}$ due to the $\mathrm{NH}$ group. 


\subsection{In Vitro Cytotoxicity Assay}

The effect of compound 5a on cellular viability was studied using the MTT Assay. The HaCaT cells are plated and cultured in 12-well cell culture plates for $24 \mathrm{~h}$ (four plates represent the four days incubation with 5a, each plate divided into 6 wells as control and 6 wells as a test) (Figure 1).

Figure 1. Represents the MTT assay results of healthy cells HaCat cells incubated with $50 \mu \mathrm{L} 0.5 \mathrm{~mol} 5 \mathbf{a}$ compared to control one. The used concentration of 5a does not induce significant cytotoxic effect on the healthy HaCaT cells.

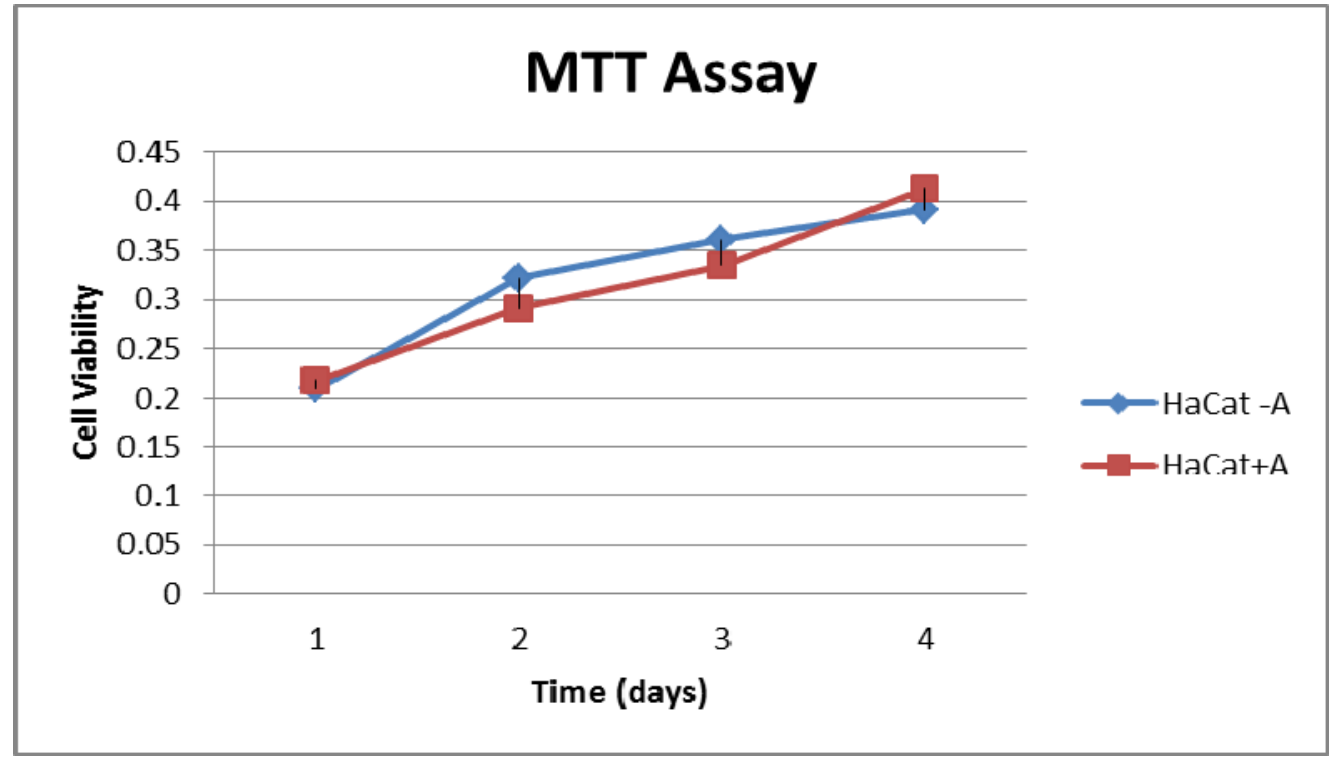

\section{Experimental}

\subsection{Chemistry}

\subsubsection{General}

Melting points were measured on an Electrothermal IA 9000 series digital melting point apparatus. The IR spectra were recorded in potassium bromide discs on a Pye Unicam SP 3300 or a Shimadzu FT IR 8101 PC infrared spectrophotometers. The NMR Spectra were recorded at $300 \mathrm{MHz}$ on a Varian Mercury VX-300 NMR spectrometer. ${ }^{1} \mathrm{H}-\mathrm{NMR}(300 \mathrm{MHz})$ and ${ }^{13} \mathrm{C}-\mathrm{NMR}(75 \mathrm{MHz})$ were run in deuterated dimethylsulphoxide (DMSO- $d_{6}$ ). Chemical shifts were related to that of the solvent. Mass spectra were recorded on a Shimadzu GCMS-QP1000 EX mass spectrometer at $70 \mathrm{eV}$. Elemental analyses of the products were carried out at the Microanalytical Centre of Cairo University, Giza, Egypt. All reactions were followed by TLC (Silica gel, Merck). Irradiation was done in an ultrasonicator, (Electric supply: 230 v, A.C. $50 \mathrm{~Hz}, 1$ phase; Ultrasonic frequency: $36 \mathrm{KHz}$; Ultrasonic power: $100 \mathrm{~W})$. In vitro cytotoxicity assay was performed at Regional Center for Food \& Feed, Agricultural Research Center, Giza, Egypt, using the MTT Assay. 2-(1-(2-oxo-2H-chromen-3yl)ethylidene)thiosemicarbazide (2) [20] and hydrazonoyl halides 3 [26,27] were prepared as reported in the literature. 
3.1.2. Synthesis of 3-[1-(4-Substituted-5-(aryldiazenyl)thiazol-2-yl)hydrazono) ethyl]-2H-chromen-2ones $\mathbf{5 a}-\mathbf{h}$

\subsubsection{Method A: General Procedure}

A mixture of 2-(1-(2-oxo-2H-chromen-3-yl)ethylidene) thiosemicarbazide (2, $0.522 \mathrm{~g}, 2 \mathrm{mmol})$ and appropriate hydrazonoyl halides $\mathbf{3}(\mathbf{4})(2 \mathrm{mmol})$ in dioxane $(30 \mathrm{~mL})$ containing triethylamine $(0.2 \mathrm{~g}$, $2 \mathrm{mmol}$ ) was irradiated by an ultrasonic generator in a water-bath at $50-60{ }^{\circ} \mathrm{C}$ for $30 \mathrm{~min}$. (monitored by TLC). The formed yellow precipitate was isolated by filtration, washed with ethanol, dried and recrystallized from dioxane to give compounds $\mathbf{5 a}-\mathbf{h}$.

3-[1-(2-(4-Methyl-5-(phenyldiazenyl)thiazol-2-yl)hydrazono)ethyl]-2H-chromen-2-one (5a). Yield $72 \%$; yellow solid; $\mathrm{mp}=142{ }^{\circ} \mathrm{C}$; IR $(\mathrm{KBr}): v 1559(\mathrm{~N}=\mathrm{N}), 1624(\mathrm{C}=\mathrm{N}), 1724(\mathrm{C}=\mathrm{O}), 3408(\mathrm{NH}) \mathrm{cm}^{-1}$; ${ }^{1} \mathrm{H}-\mathrm{NMR}\left(\mathrm{DMSO}-d_{6}\right): \delta 2.20\left(\mathrm{~s}, 3 \mathrm{H}, \mathrm{CH}_{3}\right), 2.38\left(\mathrm{~s}, 3 \mathrm{H}, \mathrm{CH}_{3}\right), 7.12-7.89(\mathrm{~m}, 9 \mathrm{H}, \mathrm{ArH}), 8.37(\mathrm{~s}, 1 \mathrm{H}$, Coumarin- $\left.\mathrm{H}_{4}\right), 10.60\left(\mathrm{~s}, 1 \mathrm{H}, \mathrm{D}_{2} \mathrm{O}\right.$ exchangeable, $\left.\mathrm{NH}\right) ;{ }^{13} \mathrm{C}-\mathrm{NMR}\left(\mathrm{DMSO}-d_{6}\right): \delta 14.61\left(\mathrm{CH}_{3}\right), 9.52$ $\left(\mathrm{CH}_{3}\right), 112.15,114.45,120.22,120.55,121.85,123.25,124.33,125.75,127.16,127.31,129.73$, 131.61, 133.73, 138.61 (Ar-C and Ar-CH), 153.13(C=N), 157.32(C=N), $167.51(\mathrm{C}=\mathrm{O}) ; \mathrm{MS} \mathrm{m} / \mathrm{z}$ (\%):404 ( $\left.\mathrm{M}^{+}+1,7\right), 403\left(\mathrm{M}^{+}, 100\right), 106$ (75), 91 (82), 77 (27). Anal. Calcd for $\mathrm{C}_{21} \mathrm{H}_{17} \mathrm{~N}_{5} \mathrm{O}_{2} \mathrm{~S}(403.11)$ : $\mathrm{C}$, $62.52 ; \mathrm{H}, 4.25 ; \mathrm{N}, 17.36$. Found $\mathrm{C}, 62.42 ; \mathrm{H}, 4.18 ; \mathrm{N}, 17.12 \%$.

3-[1-(2-(4-Methyl-5-(p-tolyldiazenyl)thiazol-2-yl)hydrazono)ethyl]-2H-chromen-2-one (5b). Yield 74\%; yellow solid; $\mathrm{mp}=230{ }^{\circ} \mathrm{C}$. IR $(\mathrm{KBr}): v 1558(\mathrm{~N}=\mathrm{N}), 1623(\mathrm{C}=\mathrm{N}), 1724(\mathrm{C}=\mathrm{O}), 3408(\mathrm{NH}) \mathrm{cm}^{-1}$; ${ }^{1} \mathrm{H}-\mathrm{NMR}\left(\mathrm{DMSO}-d_{6}\right): \delta 2.20\left(\mathrm{~s}, 3 \mathrm{H}, \mathrm{CH}_{3}\right), 2.38\left(\mathrm{~s}, 3 \mathrm{H}, \mathrm{CH}_{3}\right), 2.58\left(\mathrm{~s}, 3 \mathrm{H}, \mathrm{CH}_{3}\right), 7.12-7.88(\mathrm{~m}, 8 \mathrm{H}, \mathrm{ArH})$, $8.35\left(\mathrm{~s}, 1 \mathrm{H}\right.$, coumarin- $\left.\mathrm{H}_{4}\right), 10.63\left(\mathrm{~s}, 1 \mathrm{H}, \mathrm{D}_{2} \mathrm{O}\right.$ exchangeable, $\left.\mathrm{NH}\right) ; \mathrm{MS} m / z(\%): 417\left(\mathrm{M}^{+}, 4\right), 106$ (100), 91 (82), 65 (27). Anal. Calcd for $\mathrm{C}_{22} \mathrm{H}_{19} \mathrm{~N}_{5} \mathrm{O}_{2} \mathrm{~S}$ (417.13): C, 63.29; H, 4.59; N, 16.78. Found C, 63.22; H, 4.47; N, 16.53\%.

3-[1-(2-(5-((4-Chlorophenyl)diazenyl)-4-methylthiazol-2-yl)hydrazono)ethyl]-2H-chromen-2-one (5c). Yield 76\%; yellow solid; mp = $188^{\circ} \mathrm{C}$; IR $(\mathrm{KBr}): v 1564(\mathrm{~N}=\mathrm{N}), 1626(\mathrm{C}=\mathrm{N}), 1732(\mathrm{C}=\mathrm{O}), 3410(\mathrm{NH})$ $\mathrm{cm}^{-1} ;{ }^{1} \mathrm{H}-\mathrm{NMR}$ (DMSO-d $\left.)_{6}\right): \delta 2.20\left(\mathrm{~s}, 3 \mathrm{H}, \mathrm{CH}_{3}\right), 2.38\left(\mathrm{~s}, 3 \mathrm{H}, \mathrm{CH}_{3}\right), 7.10-7.98(\mathrm{~m}, 8 \mathrm{H}, \mathrm{ArH}), 8.38$ $\left(\mathrm{s}, 1 \mathrm{H}\right.$, coumarin- $\left.\mathrm{H}_{4}\right), 10.66\left(\mathrm{~s}, 1 \mathrm{H}, \mathrm{D}_{2} \mathrm{O}\right.$ exchangeable, $\left.\mathrm{NH}\right)$; $\mathrm{MS} m / z(\%): 438\left(\mathrm{M}^{+}+1,8\right), 437\left(\mathrm{M}^{+}, 16\right)$, 127 (100), 73 (68). Anal. Calcd for $\mathrm{C}_{21} \mathrm{H}_{16} \mathrm{ClN}_{5} \mathrm{O}_{2} \mathrm{~S}$ (437.07): C, 57.60; H, 3.68; N, 15.99. Found C, $57.51 ; \mathrm{H}, 3.62 ; \mathrm{N}, 15.71 \%$.

3-[1-(2-(4-Methyl-5-((4-nitrophenyl)diazenyl)thiazol-2-yl)hydrazono)ethyl]-2H-chromen-2-one (5d). Yield 73\%; yellow solid; $\mathrm{mp}=195{ }^{\circ} \mathrm{C}$; IR $(\mathrm{KBr}): v 1568(\mathrm{~N}=\mathrm{N}), 1629(\mathrm{C}=\mathrm{N}), 1702(\mathrm{C}=\mathrm{O}), 3404(\mathrm{NH})$ $\mathrm{cm}^{-1} ;{ }^{1} \mathrm{H}-\mathrm{NMR}\left(\mathrm{DMSO}-d_{6}\right): \delta 2.22\left(\mathrm{~s}, 3 \mathrm{H}, \mathrm{CH}_{3}\right), 2.40\left(\mathrm{~s}, 3 \mathrm{H}, \mathrm{CH}_{3}\right), 7.12-7.95(\mathrm{~m}, 8 \mathrm{H}, \mathrm{ArH}), 8.39$ (s, $1 \mathrm{H}$, coumarin- $\left.\mathrm{H}_{4}\right), 10.68\left(\mathrm{~s}, 1 \mathrm{H}, \mathrm{D}_{2} \mathrm{O}\right.$ exchangeable, $\left.\mathrm{NH}\right) ; \mathrm{MS} \mathrm{m} / \mathrm{z}(\%): 448\left(\mathrm{M}^{+}, 13\right), 201(54), 127$ (100), 82 (94), 67 (100). Anal. Calcd for $\mathrm{C}_{21} \mathrm{H}_{16} \mathrm{~N}_{6} \mathrm{O}_{4} \mathrm{~S}$ (448.10): C, 56.24; H, 3.60; N, 18.74. Found C, $56.14 ; \mathrm{H}, 3.65 ; \mathrm{N}, 18.42 \%$.

3-[1-(2-(4-Phenyl-5-(phenyldiazenyl)thiazol-2-yl)hydrazono)ethyl]-2H-chromen-2-one (5e). Yield $72 \%$; yellow solid; $\mathrm{mp}=182{ }^{\circ} \mathrm{C}$; IR $(\mathrm{KBr}): v 1558(\mathrm{~N}=\mathrm{N}), 1626(\mathrm{C}=\mathrm{N}), 1744(\mathrm{C}=\mathrm{O}), 3413(\mathrm{NH}) \mathrm{cm}^{-1}$; ${ }^{1} \mathrm{H}-\mathrm{NMR}\left(\mathrm{DMSO}-d_{6}\right): \delta 2.44\left(\mathrm{~s}, 3 \mathrm{H}, \mathrm{CH}_{3}\right), 7.12-7.95(\mathrm{~m}, 14 \mathrm{H}, \mathrm{ArH}), 8.30\left(\mathrm{~s}, 1 \mathrm{H}\right.$, coumarin- $\left.\mathrm{H}_{4}\right), 10.56$ 
(s, $1 \mathrm{H}, \mathrm{D}_{2} \mathrm{O}$ exchangeable, $\left.\mathrm{NH}\right) ;{ }^{13} \mathrm{C}-\mathrm{NMR}$ (DMSO- $\left.d_{6}\right): \delta 10.31\left(\mathrm{CH}_{3}\right), 112.35,115.35,119.43,119.55$, $121.35,123.29$, 123.52, 124.26, 125.12, 125.65, 126.34, 127.22, 129.28, 130.29, 132.33, 132.29, 136.67, 138.34 (Ar-C and Ar-CH), $154.27(\mathrm{C}=\mathrm{N}), 155.98(\mathrm{C}=\mathrm{N}), 167.29(\mathrm{C}=\mathrm{O}) ; \mathrm{MS} m / z(\%): 465$ $\left(\mathrm{M}^{+}, 10\right), 359$ (100), 134 (83), 89 (60), 77 (31). Anal. Calcd for $\mathrm{C}_{26} \mathrm{H}_{19} \mathrm{~N}_{5} \mathrm{O}_{2} \mathrm{~S}$ (465.13): C, 67.08; H, $4.11 ; \mathrm{N}, 15.04$. Found C, 67.00; H, 4.02; N, 14.86\%.

3-[1-(2-(4-Phenyl-5-(p-tolyldiazenyl)thiazol-2-yl)hydrazono)ethyl]-2H-chromen-2-one (5f). Yield 72\%; yellow solid; $\mathrm{mp}=178^{\circ} \mathrm{C}$; IR $(\mathrm{KBr}): v 1562(\mathrm{~N}=\mathrm{N}), 1628(\mathrm{C}=\mathrm{N}), 1743(\mathrm{C}=\mathrm{O}), 3415(\mathrm{NH}) \mathrm{cm}^{-1}$; ${ }^{1} \mathrm{H}-\mathrm{NMR}\left(\mathrm{DMSO}-d_{6}\right): \delta 2.20\left(\mathrm{~s}, 3 \mathrm{H}, \mathrm{CH}_{3}\right), 2.44\left(\mathrm{~s}, 3 \mathrm{H}, \mathrm{CH}_{3}\right), 7.12-7.95(\mathrm{~m}, 13 \mathrm{H}, \mathrm{ArH}), 8.31(\mathrm{~s}, 1 \mathrm{H}$, coumarin- $\left.\mathrm{H}_{4}\right), 10.59$ (s, $1 \mathrm{H}, \mathrm{D}_{2} \mathrm{O}$ exchangeable, $\left.\mathrm{NH}\right)$; $\mathrm{MS} m / z(\%): 480\left(\mathrm{M}^{+}+1,7\right), 479\left(\mathrm{M}^{+}, 100\right)$, 134 (83), 89 (82), 77 (31). Anal. Calcd for $\mathrm{C}_{27} \mathrm{H}_{21} \mathrm{~N}_{5} \mathrm{O}_{2} \mathrm{~S}$ (479.14): C, 67.62; H, 4.41; N, 14.60. Found C, $67.42 ; \mathrm{H}, 4.51 ; \mathrm{N}, 14.54 \%$.

3-[1-(2-(5-((4-Chlorophenyl)diazenyl)-4-phenylthiazol-2-yl)hydrazono)ethyl]-2H-chromen-2-one (5g). Yield 72\%; yellow solid; mp = $187^{\circ} \mathrm{C}$; IR $(\mathrm{KBr}): v 1565(\mathrm{~N}=\mathrm{N}), 1626(\mathrm{C}=\mathrm{N}), 1742(\mathrm{C}=\mathrm{O}), 3412(\mathrm{NH})$ $\mathrm{cm}^{-1}{ }^{1} \mathrm{H}-\mathrm{NMR}$ (DMSO- $\left.d_{6}\right): \delta 2.44\left(\mathrm{~s}, 3 \mathrm{H}, \mathrm{CH}_{3}\right), 7.02-7.98(\mathrm{~m}, 13 \mathrm{H}, \mathrm{ArH}), 8.34\left(\mathrm{~s}, 1 \mathrm{H}\right.$, coumarin- $\left.\mathrm{H}_{4}\right)$, 10.65 (s, 1H, $\mathrm{D}_{2} \mathrm{O}$ exchangeable, $\mathrm{NH} ; \mathrm{MS} m / z(\%): 500\left(\mathrm{M}^{+}+1,8\right), 499$ ( $\left.\mathrm{M}^{+}, 28\right), 359$ (100), 134 (64), 77 (18). Anal. Calcd for $\mathrm{C}_{26} \mathrm{H}_{18} \mathrm{ClN}_{5} \mathrm{O}_{2} \mathrm{~S}$ (499.09): C, 62.46; H, 3.63; N, 14.01. Found C, 62.44; H, $3.56 ; \mathrm{N}, 13.91 \%$.

3-[1-(2-(5-((4-Nitrophenyl)diazenyl)-4-phenylthiazol-2-yl)hydrazono)ethyl]-2H-chromen-2-one (5h). Yield 72\%; brown solid; mp = $178{ }^{\circ} \mathrm{C}$; IR $(\mathrm{KBr}): v 1566(\mathrm{~N}=\mathrm{N}), 1628(\mathrm{C}=\mathrm{N}), 1742(\mathrm{C}=\mathrm{O}), 3414(\mathrm{NH})$ $\mathrm{cm}^{-1}$; ${ }^{1} \mathrm{H}-\mathrm{NMR}$ (DMSO-d $\left.d_{6}\right): \delta 2.28\left(\mathrm{~s}, 3 \mathrm{H}, \mathrm{CH}_{3}\right), 7.12-7.95(\mathrm{~m}, 13 \mathrm{H}, \mathrm{ArH}), 8.34\left(\mathrm{~s}, 1 \mathrm{H}\right.$, coumarin- $\left.\mathrm{H}_{4}\right)$, 10.69 (s, 1H, $\mathrm{D}_{2} \mathrm{O}$ exchangeable, $\left.\mathrm{NH}\right)$; $\mathrm{MS} m / z(\%): 511\left(\mathrm{M}^{+}+1,14\right), 510\left(\mathrm{M}^{+}, 46\right), 417$ (65), 257 (24), 107 (1), 67 (100). Anal. Calcd for $\mathrm{C}_{26} \mathrm{H}_{18} \mathrm{~N}_{6} \mathrm{O}_{4} \mathrm{~S}$ (510.11): C, 61.17; H, 3.55; N, 16.46. Found C, 61.11; H, 3.48; N, 16.34\%.

\subsubsection{Method B}

\subsection{Synthesis of 3-[1-((4-Substituted thiazol-2-yl)hydrazono)ethyl]-2H-chromen-2-ones 8,9}

A mixture of $2(0.27 \mathrm{~g}, 1 \mathrm{mmol})$ and chloroacetone (6) or phenacyl bromide (7) (1 mmol) in absolute ethanol $(30 \mathrm{~mL})$ was irradiated with an ultrasonic generator in a water-bath at $50-60{ }^{\circ} \mathrm{C}$ for 20 min. (monitored by TLC). The product started to separate out during the course of reaction. The crystalline solid was filtered, washed with water, dried and recrystallized from DMF to give the corresponding compounds $\mathbf{8}$ and $\mathbf{9}$, respectively.

3-[1-(4-Methylthiazol-2-yl)hydrazono)ethyl]-2H-chromen-2-one (8). Yield 79\%; yellow solid; $\mathrm{mp}=170{ }^{\circ} \mathrm{C}$; IR (KBr): $v 1721(\mathrm{C}=\mathrm{O}), 3267(\mathrm{NH}) \mathrm{cm}^{-1} ;{ }^{1} \mathrm{H}-\mathrm{NMR}\left(\mathrm{DMSO}-d_{6}\right): \delta 2.24\left(\mathrm{~s}, 3 \mathrm{H}, \mathrm{CH}_{3}\right)$, $2.51\left(\mathrm{~s}, 3 \mathrm{H}, \mathrm{CH}_{3}\right), 7.07-8.02\left(\mathrm{~m}, 5 \mathrm{H}, \mathrm{ArH}\right.$ and thiazole- $\left.\mathrm{H}_{5}\right), 8.22\left(\mathrm{~s}, 1 \mathrm{H}\right.$, coumarin- $\left.\mathrm{H}_{4}\right), 9.31(\mathrm{~s}, 1 \mathrm{H}$, $\mathrm{D}_{2} \mathrm{O}$ exchangeable, $\left.\mathrm{NH}\right)$; MS m/z (\%): $299\left(\mathrm{M}^{+}, 6\right), 129$ (29), 77 (12), 60 (100). Anal. Calcd for $\mathrm{C}_{15} \mathrm{H}_{13} \mathrm{~N}_{3} \mathrm{O}_{2} \mathrm{~S}$ (299.07): C, 60.18; H, 4.38; N, 14.04. Found C, 60.10; H, 4.28; N, 13.84\%. 
3-[1-((4-Phenylthiazol-2-yl)hydrazono)ethyl]-2H-chromen-2-one (9). Yield 84\%; yellow solid; $\mathrm{mp}=232{ }^{\circ} \mathrm{C}$; IR $(\mathrm{KBr}): v 1744(\mathrm{C}=\mathrm{O}), 3418(\mathrm{NH}) \mathrm{cm}^{-1} ;{ }^{1} \mathrm{H}-\mathrm{NMR}\left(\mathrm{DMSO}-d_{6}\right): \delta 2.44\left(\mathrm{~s}, 3 \mathrm{H}, \mathrm{CH}_{3}\right)$, 7.10-8.02 (m, 10H, ArH and thiazole- $\left.\mathrm{H}_{5}\right), 8.26\left(\mathrm{~s}, 1 \mathrm{H}\right.$, coumarin- $\left.\mathrm{H}_{4}\right), 9.28\left(\mathrm{~s}, 1 \mathrm{H}, \mathrm{D}_{2} \mathrm{O}\right.$ exchangeable, $\mathrm{NH}) ;{ }^{13} \mathrm{C}-\mathrm{NMR}$ (DMSO- $\left.d_{6}\right): \delta 10.11\left(\mathrm{CH}_{3}\right), 112.11,116.28,119.42,120.36,122.29,123.01,124.33$, 125.75, 127.16, 127.31, 129.73, 134.33, 135.64, $139.83($ Ar-C and Ar-CH), $154.24(\mathrm{C}=\mathrm{N}), 158.12$ $(\mathrm{C}=\mathrm{N}), 167.21(\mathrm{C}=\mathrm{O})$; MS m/z (\%): $362\left(\mathrm{M}^{+}+1,12\right), 361\left(\mathrm{M}^{+}, 48\right), 359$ (100), 133 (61), 89 (81) 77 (32). Anal. Calcd for $\mathrm{C}_{20} \mathrm{H}_{15} \mathrm{~N}_{3} \mathrm{O}_{2} \mathrm{~S}$ (361.09): C, 66.46; H, 4.18; N, 11.63. Found C, C, 66.53; H, 4.10; $\mathrm{N}, 11.43 \%$.

\subsection{Coupling of 8(9) with Arenediazonium Chlorides}

To a solution of 8 or $9(1 \mathrm{mmol})$ in ethanol $(20 \mathrm{~mL})$ was added sodium acetate trihydrate $(0.138 \mathrm{~g}$, $1 \mathrm{mmol}$ ), and the mixture was cooled to $0-5{ }^{\circ} \mathrm{C}$ in an ice bath. To the resulting cold solution was added portionwise a cold solution of arenediazonium chloride [prepared by diazotizing aniline derivatives $(1 \mathrm{mmol})$ dissolved in hydrochloric acid $(6 \mathrm{M}, 1 \mathrm{~mL})$ with a solution of sodium nitrite $(0.07 \mathrm{~g}, 1 \mathrm{mmol})$ in water $(2 \mathrm{~mL})]$. After complete addition of the diazonium salt, the reaction mixture was stirred for a further $30 \mathrm{~min}$ in an ice bath. The solid that separated was filtered off, washed with water and finally recrystallized from ethanol to give product proved to be identical in all respects ( $\mathrm{mp}$, mixed mp and IR spectra) with compounds $\mathbf{5 a}-\mathbf{h}$ which obtained from method A.

3.1.3. Synthesis of 5-Arylidene-2-(2-(1-(2-oxo-2H-chromen-3-yl)ethylidene)hydrazinyl)thiazol-4(5H)ones 10a-d

\subsubsection{Method A}

A mixture of 2 (0.261 g, $1 \mathrm{mmol})$, chloroacetic acid $(0.1 \mathrm{~g}, 1 \mathrm{mmol})$ and appropriate aldehyde (1 $\mathrm{mmol})$ in glacial acetic acid $(20 \mathrm{~mL})$ containing anhydrous sodium acetate $(0.33 \mathrm{~g}, 4 \mathrm{mmol})$ was irradiated with an ultrasonic generator in a water-bath at $50-60{ }^{\circ} \mathrm{C}$ for $30 \mathrm{~min}$. (monitored by TLC). The reaction mixture was left to cool and the formed solid was filtered off, washed with water, dried and recrystallized from ethanol to give $\mathbf{1 0 a}-\mathbf{d}$.

5-Benzylidene-2-[2-(1-(2-oxo-2H-chromen-3-yl)ethylidene)hydrazinyl]thiazol-4(5H)-one (10a). Yield 84\%; yellow solid; $\mathrm{mp}=176{ }^{\circ} \mathrm{C}$; IR $(\mathrm{KBr}): v 1678,1725(2 \mathrm{C}=\mathrm{O}), 3360(\mathrm{NH}) \mathrm{cm}^{-1}$; ${ }^{1} \mathrm{H}-\mathrm{NMR}\left(\mathrm{DMSO}-d_{6}\right)$ : $\delta 2.24\left(\mathrm{~s}, 3 \mathrm{H}, \mathrm{CH}_{3}\right), 6.91-7.88(\mathrm{~m}, 9 \mathrm{H}, \mathrm{ArH}), 8.19\left(\mathrm{~s}, 1 \mathrm{H}\right.$, coumarin- $\left.\mathrm{H}_{4}\right), 8.63(\mathrm{~s}, 1 \mathrm{H}, \mathrm{N}=\mathrm{CH}), 10.87$ (s, $1 \mathrm{H}, \mathrm{D}_{2} \mathrm{O}$ exchangeable, $\left.\mathrm{NH}\right) ;{ }^{13} \mathrm{C}-\mathrm{NMR}$ (DMSO- $\left.d_{6}\right): \delta 9.97\left(\mathrm{CH}_{3}\right), 112.15,114.45,121.23,121.62$, $121.89,122.42,124.19,124.84,126.46,128.43,129.87,130.41,134.43,146.73$ (Ar-C and Ar-CH), $154.19(\mathrm{C}=\mathrm{N}), 158.12(\mathrm{C}=\mathrm{N}), 167.11(\mathrm{C}=\mathrm{O}), 181.56(\mathrm{C}=\mathrm{O}) ; \mathrm{MS} m / z(\%): 302\left(\mathrm{M}^{+}+1,9\right), 301$ $\left(\mathrm{M}^{+}, 62\right), 218$ (65), 172 (40), 130 (100), 77 (43). Anal. Calcd for $\mathrm{C}_{21} \mathrm{H}_{15} \mathrm{~N}_{3} \mathrm{O}_{3} \mathrm{~S}$ (389.08): C, 64.77; H, $3.88 ; \mathrm{N}, 10.79$. Found C, 64.58; H, 3.78; N, 10.48\%.

5-[4-Methylbenzylidene)-2-(2-(1-(2-oxo-2H-chromen-3-yl)ethylidene)hydrazinyl)thiazol-4(5H)-one (10b). Yield 79\%; yellow solid; $\mathrm{mp}=172{ }^{\circ} \mathrm{C}$; IR $(\mathrm{KBr}): v 1678,1721(2 \mathrm{C}=\mathrm{O}), 3353(\mathrm{NH}) \mathrm{cm}^{-1}$; ${ }^{1} \mathrm{H}-\mathrm{NMR}$ (DMSO-d $\left.d_{6}\right): \delta 2.24\left(\mathrm{~s}, 3 \mathrm{H}, \mathrm{CH}_{3}\right), 2.51\left(\mathrm{~s}, 3 \mathrm{H}, \mathrm{CH}_{3}\right), 6.91-7.88(\mathrm{~m}, 8 \mathrm{H}, \mathrm{ArH}), 8.19\left(\mathrm{~s}, 1 \mathrm{H}\right.$, coumarin- $\left.\mathrm{H}_{4}\right)$, $8.60(\mathrm{~s}, 1 \mathrm{H}, \mathrm{N}=\mathrm{CH}), 10.83\left(\mathrm{~s}, 1 \mathrm{H}, \mathrm{D}_{2} \mathrm{O}\right.$ exchangeable, $\left.\mathrm{NH}\right) ; \mathrm{MS} m / z(\%): 404\left(\mathrm{M}^{+}+1,15\right), 403$ 
$\left(\mathrm{M}^{+}, 46\right), 235$ (100), 146 (64), 130 (18), 77 (39). Anal. Calcd for $\mathrm{C}_{22} \mathrm{H}_{17} \mathrm{~N}_{3} \mathrm{O}_{3} \mathrm{~S}$ (403.10): C, 65.49; $\mathrm{H}$, $4.25 ; \mathrm{N}, 10.42$. Found $\mathrm{C}, 65.43 ; \mathrm{H}, 4.11 ; \mathrm{N}, 10.12 \%$.

5-(4-Chlorobenzylidene)-2-[2-(1-(2-oxo-2H-chromen-3-yl)ethylidene)hydrazinyl]thiazol-4(5H)-one (10c). Yield 82\%; yellow solid; $\mathrm{mp}=186{ }^{\circ} \mathrm{C}$; IR $(\mathrm{KBr}): v 1679,1722(2 \mathrm{C}=\mathrm{O}), 3353(\mathrm{NH}) \mathrm{cm}^{-1}$; ${ }^{1} \mathrm{H}-\mathrm{NMR}$ $\left(\mathrm{DMSO}-d_{6}\right): \delta 2.26\left(\mathrm{~s}, 3 \mathrm{H}, \mathrm{CH}_{3}\right), 6.91-7.96(\mathrm{~m}, 8 \mathrm{H}, \mathrm{ArH}), 8.19\left(\mathrm{~s}, 1 \mathrm{H}\right.$, coumarin- $\left.\mathrm{H}_{4}\right), 8.62(\mathrm{~s}, 1 \mathrm{H}$, $\mathrm{N}=\mathrm{CH}), 10.86\left(\mathrm{~s}, 1 \mathrm{H}, \mathrm{D}_{2} \mathrm{O}\right.$ exchangeable, $\left.\mathrm{NH}\right) ; \mathrm{MS} m / z(\%): 424\left(\mathrm{M}^{+}+1,13\right), 423\left(\mathrm{M}^{+}, 46\right), 235$ (100), 172 (36), 130 (58), 63 (69). Anal. Calcd for $\mathrm{C}_{21} \mathrm{H}_{14} \mathrm{ClN}_{3} \mathrm{O}_{3} \mathrm{~S}$ (423.04): C, 59.50; H, 3.33; N, 9.91. Found C, 59.37; H, 3.13; N, 9.61\%.

5-(4-Nitrobenzylidene)-2-[2-(1-(2-oxo-2H-chromen-3-yl)ethylidene)hydrazinyl]thiazol-4(5H)-one (10d). Yield 76\%; yellow solid; $\mathrm{mp}=170{ }^{\circ} \mathrm{C}$; IR $(\mathrm{KBr}): v 1679,1723(2 \mathrm{C}=\mathrm{O}), 3366(\mathrm{NH}) \mathrm{cm}^{-1}$; ${ }^{1} \mathrm{H}-\mathrm{NMR}$ $\left(\right.$ DMSO- $\left.d_{6}\right): \delta 2.26\left(\mathrm{~s}, 3 \mathrm{H}, \mathrm{CH}_{3}\right), 6.91-7.96(\mathrm{~m}, 8 \mathrm{H}, \mathrm{ArH}), 8.16\left(\mathrm{~s}, 1 \mathrm{H}\right.$, coumarin $\left.-\mathrm{H}_{4}\right), 8.67(\mathrm{~s}, 1 \mathrm{H}$, $\mathrm{N}=\mathrm{CH}), 10.86\left(\mathrm{~s}, 1 \mathrm{H}, \mathrm{D}_{2} \mathrm{O}\right.$ exchangeable, $\left.\mathrm{NH}\right) ; \mathrm{MS} m / z(\%): 435\left(\mathrm{M}^{+}+1,7\right), 434\left(\mathrm{M}^{+}, 44\right), 235(100)$, 218 (66), 130 (70), 63 (58). Anal. Calcd for $\mathrm{C}_{21} \mathrm{H}_{14} \mathrm{~N}_{4} \mathrm{O}_{5} \mathrm{~S}$ (434.07): C, 58.06; H, 3.25; N, 12.90. Found C, 57.86; H, 3.26; N, $12.79 \%$.

\subsubsection{Method B}

3.1.3.2.1. Synthesis of 2-[2-(1-(2-oxo-2H-chromen-3-yl)ethylidene)hydrazinyl]thiazol-4(5H)-one (11)

A mixture of $2(0.261 \mathrm{~g}, 1 \mathrm{mmol})$ and chloroacetic acid $(0.1 \mathrm{~g}, 1 \mathrm{mmol})$ in glacial acetic acid $(30 \mathrm{~mL})$ containing anhydrous sodium acetate $(0.33 \mathrm{~g}, 4 \mathrm{mmol})$ was irradiated with an ultrasonic generator in a water-bath at $50-60{ }^{\circ} \mathrm{C}$ for $30 \mathrm{~min}$. (monitored by TLC). The reaction mixture was cooled and the resulting precipitate was filtered off and recrystallized from ethanol to give 11. Yield 84\%; yellow solid; $\mathrm{mp}=220^{\circ} \mathrm{C}$; IR $(\mathrm{KBr}): v 1667,1717(2 \mathrm{C}=\mathrm{O}), 3167(\mathrm{NH}) \mathrm{cm}^{-1}$; ${ }^{1} \mathrm{H}-\mathrm{NMR}\left(\mathrm{DMSO}-d_{6}\right)$ : $\delta 2.26\left(\mathrm{~s}, 3 \mathrm{H}, \mathrm{CH}_{3}\right), 3.97\left(\mathrm{~s}, 2 \mathrm{H}, \mathrm{CH}_{2}\right), 6.91-8.19(\mathrm{~m}, 4 \mathrm{H}, \mathrm{ArH}), 8.63$ (s, 1H, coumarin- $\left.\mathrm{H}_{4}\right), 10.87$ (s, $1 \mathrm{H}, \mathrm{D}_{2} \mathrm{O}$ exchangeable, $\left.\mathrm{NH}\right) ;{ }^{13} \mathrm{C}-\mathrm{NMR}\left(\mathrm{DMSO}-d_{6}\right): \delta 10.46\left(\mathrm{CH}_{3}\right), 39.35\left(\mathrm{CH}_{2}\right), 118.45,120.18$, 122.23, 124.33, 129.73, 131.61, 133.73, $138.61(\mathrm{Ar}-\mathrm{C}$ and $\mathrm{Ar}-\mathrm{CH}), 153.43(\mathrm{C}=\mathrm{N}), 156.56(\mathrm{C}=\mathrm{N})$, $167.12(\mathrm{C}=\mathrm{O}), 176.33(\mathrm{C}=\mathrm{O})$; $\mathrm{MS} m / z(\%): 303\left(\mathrm{M}^{+}+2,3\right), 302\left(\mathrm{M}^{+}+1,9\right), 301\left(\mathrm{M}^{+}, 36\right), 235(73)$, 146 (36), 130 (100), 77 (59). Anal. Calcd for $\mathrm{C}_{14} \mathrm{H}_{11} \mathrm{~N}_{3} \mathrm{O}_{3} \mathrm{~S}$ (301.05): C, 55.80; H, 3.68; N, 13.95. Found C, 55.68; H, 3.60; N, 13.75\%.

\subsection{Reaction of $\mathbf{8}$ with Aromatic Aldehydes}

General procedure: To a solution of 5-thiazolidinone $11(0.30 \mathrm{~g}, 1 \mathrm{mmol})$ and appropriate aldehyde $(1 \mathrm{mmol})$ in glacial acetic acid $(20 \mathrm{~mL})$, anhydrous sodium acetate $(0.33 \mathrm{~g}, 4 \mathrm{mmol})$ was irradiated with an ultrasonic generator in a water-bath at $50-60{ }^{\circ} \mathrm{C}$ for $30 \mathrm{~min}$ (monitored by TLC). The product, so separated, was filtered, washed with water, dried and recrystallized from ethanol to give compounds which proved to be identical in all respects (mp, mixed $\mathrm{mp}$ and IR spectra) with the hydrazonothiazolidinones $\mathbf{1 0 a}-\mathbf{d}$ which obtained from method A. 


\subsubsection{Synthesis of 2-Cyano- $N^{\prime}-(1-(2-o x o-2 H$-chromen-3-yl)ethylidene)acetohydrazide (12)}

To a solution of 2-cyanoacetohydrazide $(1.0 \mathrm{~g}, 10 \mathrm{mmol})$ and 3 -acetyl- $2 H$-chromen-2-one $(\mathbf{1}, 1.88 \mathrm{~g}$, $10 \mathrm{mmol})$ in absolute ethanol $(30 \mathrm{~mL})$ three drops of conc. $\mathrm{HCl}$ were added and the reaction mixture was irradiated with an ultrasonic generator in a water-bath at $50-60{ }^{\circ} \mathrm{C}$ for $20 \mathrm{~min}$. then left to cool. The solid product formed was collected by filtration, dried and recrystallized from ethanol to give 12. Yield 86\%; yellow microcrystals; $\mathrm{mp}=172{ }^{\circ} \mathrm{C}$; IR $(\mathrm{KBr}): v=1690,1724(2 \mathrm{C}=\mathrm{O}), 2230(\mathrm{CN}), 3186$ $(\mathrm{NH}) \mathrm{cm}^{-1}$; ${ }^{1} \mathrm{H}-\mathrm{NMR}$ (DMSO-d $): \delta 2.17\left(\mathrm{~s}, 3 \mathrm{H}, \mathrm{CH}_{3}\right), 4.25\left(\mathrm{~s}, 2 \mathrm{H}, \mathrm{CH}_{2}\right), 7.39-8.30(\mathrm{~m}, 4 \mathrm{H}, \mathrm{ArH})$, $8.95\left(\mathrm{~s}, 1 \mathrm{H}\right.$, coumarin- $\left.\mathrm{H}_{4}\right), 11.19\left(\mathrm{~s}, 1 \mathrm{H}, \mathrm{D}_{2} \mathrm{O}\right.$ exchangeable, $\left.\mathrm{NH}\right)$; $\mathrm{MS} m / z(\%): 270\left(\mathrm{M}^{+}+1,5\right), 269$ $\left(\mathrm{M}^{+}, 33\right), 229$ (82), 115 (100), 89 (60), 63 (54). Anal. Calcd for $\mathrm{C}_{14} \mathrm{H}_{11} \mathrm{~N}_{3} \mathrm{O}_{3}$ (269.08): C, 62.45; H, 4.12; N, 15.61. Found C, C, 62.41; H, 4.02; N, 15.38\%.

\subsubsection{Reaction of $\mathbf{1 1}$ with Aromatic Aldehydes}

General procedure: Equimolecular mixture of 2-cyano- $N$ '-(1-(2-oxo- $2 H$-chromen-3-yl)ethylidene) acetohydrazide $(\mathbf{1 2}, 2.69 \mathrm{~g}, 0.01 \mathrm{~mol})$ and appropriate aldehyde $(0.01 \mathrm{~mol})$, in anhydrous ethanol $(20 \mathrm{~mL})$ containing piperidine $(0.50 \mathrm{~mL})$ was irradiated with an ultrasonic generator in a water-bath at 50-60 ${ }^{\circ} \mathrm{C}$ for $30 \mathrm{~min}$ (monitored by TLC). The formed solid was collected by filtration and recrystallized from the proper solvent to give compounds $\mathbf{1 3 a}-\mathbf{d}$.

2-Cyano-N'-[1-(2-oxo-2H-chromen-3-yl)ethylidene]-3-phenylacrylohydrazide (13a). Yield 80\%; yellow solid (from ethanol); $\mathrm{mp}=198{ }^{\circ} \mathrm{C}$; IR $(\mathrm{KBr}): v$ 1672, $1728(2 \mathrm{C}=\mathrm{O}), 2210(\mathrm{CN}), 3336(\mathrm{NH}) \mathrm{cm}^{-1}$; ${ }^{1} \mathrm{H}-\mathrm{NMR}\left(\mathrm{DMSO}-d_{6}\right): \delta 2.19\left(\mathrm{~s}, 3 \mathrm{H}, \mathrm{CH}_{3}\right), 7.24-8.21(\mathrm{~m}, 9 \mathrm{H}, \mathrm{ArH}), 8.87\left(\mathrm{~s}, 1 \mathrm{H}\right.$, coumarin- $\left.\mathrm{H}_{4}\right), 9.34$ $(\mathrm{s}, 1 \mathrm{H}, \mathrm{C}=\mathrm{CH}), 11.22\left(\mathrm{~s}, 1 \mathrm{H}, \mathrm{D}_{2} \mathrm{O}\right.$ exchangeable, $\left.\mathrm{NH}\right) ;{ }^{13} \mathrm{C}-\mathrm{NMR}\left(\mathrm{DMSO}-d_{6}\right): \delta 10.74\left(\mathrm{CH}_{3}\right), 112.36$, $115.26,117.26,119.42,121.65,122.86,123.43,124.16,124.86,127.67,127.99,129.04,133.43$, 134.87, $139.37($ Ar-C, Ar- $\mathrm{CH}$ and $\mathrm{C} \equiv \mathrm{N}), 154.94(\mathrm{C}=\mathrm{N}), 168.13(\mathrm{C}=\mathrm{O}), 174.14(\mathrm{C}=\mathrm{O}) ; \mathrm{MS} \mathrm{m} / z(\%)$ : $358\left(\mathrm{M}^{+}+1,4\right), 357\left(\mathrm{M}^{+}, 40\right), 185$ (100), 129 (73), 109 (73), 55 (91). Anal. Calcd for $\mathrm{C}_{21} \mathrm{H}_{15} \mathrm{~N}_{3} \mathrm{O}_{3}$ (357.11): C, 70.58; H, 4.23; N, 11.76. Found C, 70.49; H, 4.21; N, 11.46\%.

2-Cyano-N'-[1-(2-oxo-2H-chromen-3-yl)ethylidene]-3-p-tolylacrylohydrazide (13b). Yield 82\%; yellow solid (from DMF); $\mathrm{mp}=187^{\circ} \mathrm{C}$. IR $(\mathrm{KBr}): v 1672,1728(2 \mathrm{C}=\mathrm{O}), 2216(\mathrm{CN}), 3336(\mathrm{NH}) \mathrm{cm}^{-1}$; ${ }^{1} \mathrm{H}-\mathrm{NMR}\left(\mathrm{DMSO}-d_{6}\right): \delta 2.19\left(\mathrm{~s}, 3 \mathrm{H}, \mathrm{CH}_{3}\right), 2.49$ (s, 3H, $\left.\mathrm{CH}_{3}\right), 7.20-8.23(\mathrm{~m}, 8 \mathrm{H}, \mathrm{ArH}), 8.89(\mathrm{~s}, 1 \mathrm{H}$, coumarin- $\left.\mathrm{H}_{4}\right), 9.34(\mathrm{~s}, 1 \mathrm{H}, \mathrm{C}=\mathrm{CH}), 11.26\left(\mathrm{~s}, 1 \mathrm{H}, \mathrm{D}_{2} \mathrm{O}\right.$ exchangeable, $\left.\mathrm{NH}\right) ; \mathrm{MS} m / z(\%): 372\left(\mathrm{M}^{+}+1,6\right)$, $371\left(\mathrm{M}^{+}, 45\right), 229$ (75), 186 (55), 115 (100), 63 (62). Anal. Calcd for $\mathrm{C}_{22} \mathrm{H}_{17} \mathrm{~N}_{3} \mathrm{O}_{3}$ (371.13): C, 71.15; $\mathrm{H}, 4.61 ; \mathrm{N}, 11.31$. Found $\mathrm{C}, \mathrm{C}, 71.05 ; \mathrm{H}, 4.36 ; \mathrm{N}, 11.11 \%$.

3-(4-Chlorophenyl)-2-cyano-N'-(1-(2-oxo-2H-chromen-3-yl)ethylidene)acrylohydrazide (13c). Yield $78 \%$; yellow solid (from ethanol ); $\mathrm{mp}=202{ }^{\circ} \mathrm{C}$; IR (KBr): $v$ 1674, $1732(2 \mathrm{C}=\mathrm{O}), 2218(\mathrm{CN}), 3334$ $(\mathrm{NH}) \mathrm{cm}^{-1} ;{ }^{1} \mathrm{H}-\mathrm{NMR}$ (DMSO- $\left.d_{6}\right): \delta 2.19\left(\mathrm{~s}, 3 \mathrm{H}, \mathrm{CH}_{3}\right), 7.21-8.23(\mathrm{~m}, 8 \mathrm{H}, \mathrm{ArH}), 8.89\left(\mathrm{~s}, 1 \mathrm{H}\right.$, coumarin- $\left.\mathrm{H}_{4}\right)$, 9.35 (s, 1H, C=CH), 11.34 (s, 1H, $\mathrm{D}_{2} \mathrm{O}$ exchangeable, $\left.\mathrm{NH}\right)$; $\mathrm{MS} m / z(\%): 392\left(\mathrm{M}^{+}+1,7\right), 391\left(\mathrm{M}^{+}, 22\right)$, 273 (47), 219 (100), 84(94). Anal. Calcd for $\mathrm{C}_{21} \mathrm{H}_{14} \mathrm{ClN}_{3} \mathrm{O}_{3}$ (391.07): C, 64.37; H, 3.60; N, 10.72. Found C, C, 64.07; H, 3.62; N, 10.32\%. 
2-Imino-N'-[1-(2-oxo-2H-chromen-3-yl)ethylidene]-2H-chromene-3-carbohydrazide (14). Yield 78\%; yellow solid (from DMF); $\mathrm{mp}=240{ }^{\circ} \mathrm{C}$; IR $(\mathrm{KBr}): v 1669,1712(2 \mathrm{C}=\mathrm{O}), 3198,3325(2 \mathrm{NH}) \mathrm{cm}^{-1}$; ${ }^{1} \mathrm{H}-\mathrm{NMR}$ (DMSO- $d_{6}$ ): $\delta 2.19$ (s, 3H, $\mathrm{CH}_{3}$ ), 7.10-8.36 (m, 9H, ArH ), 8.44 (s, 1H, $\mathrm{D}_{2} \mathrm{O}$ exchangeable, NH), $8.89\left(\mathrm{~s}, 1 \mathrm{H}\right.$, coumarin- $\left.\mathrm{H}_{4}\right), 11.38\left(\mathrm{~s}, 1 \mathrm{H}, \mathrm{D}_{2} \mathrm{O}\right.$ exchangeable, $\left.\mathrm{NH}\right) ; \mathrm{MS} m / z(\%): 374\left(\mathrm{M}^{+}+1,13\right), 373$ $\left(\mathrm{M}^{+}, 13\right), 201$ (51), 171 (100), 115 (89), 62 (58). Anal. Calcd for $\mathrm{C}_{21} \mathrm{H}_{15} \mathrm{~N}_{3} \mathrm{O}_{4}$ (373.11): C, 67.56; H, $4.05 ; \mathrm{N}, 11.25$. Found C, C, 67.44; H, 4.15; N, 11.05\%.

\subsection{Cytotoxic Activity}

The method applied is similar to that reported by Skehan et al. using the 3-(4,5-dimethylthiazol-2yl)-2,5-diphenyl-2H-tetrazolium bromide (MTT) assay. Healthy HaCaT epithelial primary cell line (human keratinocytes) was cultured on $18 \mathrm{~mm}$ diameter glass cover slips in a 12-well tissue culture plate in DMEM plus 5\% FBS at $37-38{ }^{\circ} \mathrm{C}$ under $5 \% \mathrm{CO}_{2}$. The cover slips were coated with collagen type I (Roche) in advance for optimum cell growth. The HaCaT cells are cultured in 12-well cell culture plates for $24 \mathrm{~h}$, (four plates represent the four days incubation with 5a, each plate divided into 6 -wells as control and 6-wells as a test). Rinsing the old medium and adding new one then $50 \mu \mathrm{L}$ $0.5 \mathrm{~mol} 5 \mathrm{a}$ are added to test wells (not to the control one) and returned to incubator for $8 \mathrm{~h}$ washing the excess 5a by DPBS buffer and add $1 \mathrm{~mL}$ medium to each well then incubated for the designated periods for each plate (Figure 1).

\section{Conclusions}

In summary, we have developed a new green methodology and synthesized several 3-[1-(4substituted-5-(aryldiazenyl)thiazol-2-yl)hydrazono)ethyl]-2H-chromen-2-ones by ultrasound irradiation. The cytototoxic activity of one of them was also evaluated against healthy HaCaT cells.

\section{Acknowledgements}

The authors wish to thank Taher Salah of Nanotechnology Center, Regional Center for Food \& Feed, Agricultural Research Center for his support to carry out the antimicrobial activity.

\section{References}

1. Czerpack, R.; Skolska, S. Effect of selected synthetic regulators on Pseudomonas aeruginosa growth in liquid culture. Med. Dosw. Microbiol. 1982, 34, 37-50.

2. Jund, L.; Corse, J.; King, A.S.; Bayne, H.; Mihrag, K. Antimicrobial properties of 6,7-dihydroxy7,8-dihydroxy-, 6-hydroxy- and 8-hydroxycoumarins. Phytochemistry 1971, 10, 2971-2974.

3. El-Ansary, S.L.; Aly, E.I.; Halem, M.A. New coumarin derivatives as antibacterial agents. Egypt J. Pharm. Sci. 1992, 33, 379-390.

4. Reddy, Y.D.; Somayojulu, V.V. Synthesis, spectra and physiological activity of 7H-pyrano[3,2c]benzoxazole-7-one. J. Ind. Chem. Soc. 1981, 58, 599-601.

5. Weber, U.S.; Steffen, B.; Siegers, C. Antitumor activities of coumarin, 7-hydroxy-coumarin and its glucuronide in several human tumor cell lines. Res. Commun. Mol. Pathol. Pharmacol. 1998, 99, 93-206. 
6. Küçükgüzel, G.; Kocatepe, A.; Clercq, E.D.; Şahin, F.; Güllüce, M. Synthesis and biological activity of 4-thiazolidinones, thiosemicarbazides derived from diflunisal hydrazide. Eur. J. Med. Chem. 2006, 41, 353-359.

7. Verma, A.; Saraf, S.K. 4-Thiazolidinone-Abiologicallyactivescaffold. Eur. J. Med. Chem. 2008, 43, 897-905.

8. Akerblom, E.B. Synthesis and structure-activity relations of a series of antibacterially active 5-(5nitro-2-furfurylidene)thiazolones, 5-(5-nitro-2-furylpropenylidene)thiazolones, and 6-(5-nitro-2furyl)-4H-1,3-thiazinones. J. Med. Chem. 1974, 17, 609-615.

9. Abdel-Wahab, B.F.; Mohamed, S.F.; Amr, A.El-G.E.; Abdalla, M.M. Synthesis and reactions of thiosemicarbazides, Triazoles, and Schiff bases as antihypertensive $\alpha$-blocking agents. Monatsh. Chem. 2008, 139, 1083-1090.

10. Amir, M.; Agarwal, R. Synthesis and antibacterial activity of 1-thiocarbamoyl-3-methyl-4arylhydrazono)-2-pyrazolin-5-one. J. Ind. Chem. Soc. 1997, 74, 154-155.

11. Gholap, A.R.; Venkatesan, K.; Daniel, T.R.; Lahoti, J.; Srinivasan, K.V. Ionic liquid promoted novel and efficient one pot synthesis of 3,4-dihydropyrimidin-2-(1H)-ones at ambient temperature under ultrasound irradiation. Green Chem. 2004, 6, 147.

12. Bazgir, A.; Ahadi, S.; Ghahremanzadeh, R.; Khavasi, H.R.; Mirzaei, P. Ultrasound-assisted onepot, three-component synthesis of spiro[indoline-3,4'-pyrazolo[3,4- $b]$ pyridine]-2,6' $\left(1^{\prime} H\right)$-diones in water. Ultrason. Sonochem. 2010, 17, 447-452.

13. Zhang, Z.H.; Li, J.J.; Li, T.S. Ultrasound-assisted synthesis of pyrroles catalyzed by zirconium chloride under solvent-free conditions. Ultrason. Sonochem. 2008, 15, 673-676.

14. Abd-el-Rahman, N.M.; Saleh, T.S.; Mady, M.F. Ultrasound assisted synthesis of some new 1,3,4-thiadiazole and bi(1,3,4-thiadiazole) derivatives incorporating pyrazolone moiety. Ultrason. Sonochem. 2009, 16, 70-74.

15. Jiang, Y.; Chen, X.; Qu, L.; Wang, J.; Yuan, J. Chen, S.; Li, X.; Qu, C. Ultrasonic-assisted synthesis of chrysin derivatives linked with 1,2,3-triazoles by 1,3-dipolar cycloaddition reaction. Ultrason. Sonochem. 2011, 18, 527-533.

16. Mahdavinia, G.H.; Rostamizadeh, S.; Amani, A.M.; Emdadi, Z. Ultrasound-promoted greener synthesis of aryl-14- $H$-dibenzo $[a, j]$ xanthenes catalyzed by $\mathrm{NH}_{4} \mathrm{H}_{2} \mathrm{PO}_{4} / \mathrm{SiO}_{2}$ in water. Ultrason. Sonochem. 2009, 16, 7-10.

17. Gomha, S.M.; Abdel-Aziz, H.A. Synthesis of new heterocycles derived from 3-(3-methyl-1Hindol-2-yl)-3-oxopropanenitrile as potent antifungal agents. Bull. Korean Chem. Soc. 2012, in press.

18. Riyadh, S.M.; Farghaly, T.A.; Gomha, S.M. Novel polyazaheterocyclic systems: Synthesis, antitumor, and antimicrobial Activities. Arch. Pharm. Res. 2010, 33, 1721-1728.

19. Gomha, S.M. ; Hassaneen, H.M.E. Synthesis and antimicrobial activity of some new pyrazoles, fused pyrazolo[3,4- $d]$-pyrimidine and 1, 2-dihydroimidazo-[2,1-c][1,2,4]triazin-6-one derivatives. Molecules 2011, 16, 6549-6560.

20. Chimenti, F.; Bolasco, A.; Secci, D.; Chimenti, P.; Granese, A.; Carradori, S.; Yáñez, M.; Orallo, F.; Ortuso, F.; Alcaro, S. Investigations on the 2-thiazolylhydrazyne scaffold: Synthesis and molecular modeling of selective human monoamine oxidase inhibitors. Bioorg. Med. Chem. 2010, 18, 5715-5723. 
21. Hantzsch, A.; Weber, J.H. Ueber Verbindungen des Thiazols (Pyridins der Thiophenreihe). Dtsch. Chem. Ges. 1887, 20, 3118-3132.

22. Metwally, M.A.; Abdel-latif, E.; Amer, F.A.; Kaupp, G. Synthesis of new 5-thiazolyl azo-disperse dyes for dyeing polyester fabrics. Dyes Pigments 2004, 60, 249-264.

23. Metwally, M.A.; Abdel-Latif, E.; Amer, F.A. New 4-arylazo-2-(substituted)-3-phenyl-1,3thiazolidin-5-ones as disperse dyes part 1. J. Text. Assoc. 2001, 63, 155-159.

24. Zhou, J.F.; Gong, G.X.; Zhu, F.X.; Zhi, S. Microwave promoted one-pot synthesis of 3-(2'-amino3'-cyano-4'-arylpyrid-6'-yl) coumarins. J. Chin. Chem. Lett. 2009, 20, 37-39.

25. Volmajer, J.; Toplak, R.; Leban, I.; LeMarechal, A.M. Synthesis of new iminocoumarins and their transformations into $N$-chloro and hydrazono compounds. Tetrahedron 2005, 61, 7012-7021.

26. Eweiss, N.F.; Osman, A. Synthesis of heterocycles. Part II. New routes to acetylthiadiazolines and alkylazothiazoles. J. Heterocycl. Chem. 1980, 17, 1713-1717.

27. Shawali, A.S.; Abdelhamid, A.O. Reaction of dimethylphenacylsulfonium bromide with $\mathrm{N}$-nitrosoacetarylamides and reactions of the products with nucleophiles. Bull. Chem. Soc. Jpn. 1976, 49, 321-327.

Sample Availability: Samples of the synthesized compounds are available from the authors.

(C) 2012 by the authors; licensee MDPI, Basel, Switzerland. This article is an open access article distributed under the terms and conditions of the Creative Commons Attribution license (http://creativecommons.org/licenses/by/3.0/). 